\section{Transmastoid Cartilage Graft-Meningocele Temporal: Surgical Approach}

\section{Mariana Dalbo Contrera Toro ${ }^{1 *}$, Alexandre Caixeta Guim- arães $^{2}$, Guilherme Machado de Carvalho' ${ }^{2}$, Jorge Rizzato Paschoal $^{3}$ and Leopoldo Nizam Pfeilsticker ${ }^{4}$}

${ }^{1}$ Department of Ear, Nose, Throat and Head \& Neck Surgery, University of Campinas, São Paulo, Brazil

${ }^{2}$ Department of Otology, Audiology and Implantable Ear Prostheses, University of Campinas, São Paulo, Brazil

${ }^{3}$ Department of ENT, Skull Base, Head of Skull base ENT Service, Campinas University, São Paulo, Brazil

${ }^{4}$ Department of ENT, Maxillofacial, Head of Maxillofacial ENT Service, São Paulo, Brazil

\section{Abstract \\ Introduction}

Meningocele of the temporal bone is a herniation of meninges located in the middle ear, mastoid, petrous apex, or posterior fossa. The pathogenesis of this disease is a combination of temporal bone defects, along with a duramater alteration. Some correction techniques are described in the literature, but there is no consensus on the best approach.

\section{Objective}

The aim of this study is to describe the surgical treatment of temporal meningocele and to review the medical literature on this topic.

\section{Methods}

Retrospective analysis of clinical records of two patients who underwent surgical treatment for temporal bone meningocele along with review of the medical literature in PubMed.

\section{Results}

Both patients underwent surgical repair of meningocele via mastoidectomy with ear cartilage graft, fibrin glue and temporal fascia.

\section{Discussion}

In the literature, eight publications reporting 135 cases of meningocele of the temporal bone were found. Transmastoid and combined access with craniotomy surgical approaches were

*Corresponding author: Mariana Dalbo Contrera Toro, Department of Ear Nose, Throat and Head \& Neck Surgery, University of Campinas, São Paulo, Brazil, Tel: +55 1935217523; Fax: +55 1935217563; E-mail: mdctoro@gmail.com

Citation: Toro MDC, Guimarães AC, de Carvalho GM, Paschoal JR, Pfeilsticker LN (2016) Transmastoid Cartilage Graft-Meningocele Temporal: Surgical Approach. J Otolaryng Head Neck Surg 2: 005.

Received: December 21, 2015; Accepted: March 03, 2016; Published: March 21,2016 reported. The use of cartilage graft, temporalis fascia and mucosal flap closure for the correction of meningocele was described.

\section{Conclusion}

Meningocele temporal bone is a condition that must be considered as a differential diagnosis of otorrhea in patients with a history of ear surgery or trauma. Surgical correction is necessary for improvement of symptoms and prevention of meningitis. The technique described in this study proved effective for correction of pathology, which can be confirmed by similar results reported in the literature.

Keywords: Cartilage graft; Meningocele temporal; Surgical techniques

\section{Introduction}

Temporal bone meningocele is a herniation of meninges into the middle ear, mastoid, petrous apex, or posterior cranial fossa through defects in the temporal bone. When there is an associated brain tissue herniation from the temporal lobe, the prolapsed tissue is then called meningoencephalocele or just encephalocele [1].

It can be divided in two groups according to the etiology: spontaneous or acquired. The most common kind is acquired, usually resulting from otologic infections, neoplasia, otologic surgery or head trauma. Spontaneous meningoencephalocele may occur mostly in the epitympanum or mastoid antrum and it can also be a result of pathologic obesity [2].

The pathogenesis of this disease results from a combination of bone defects along with an alteration of the duramater, that allows the herniation of brain and meningeal tissue, which explains why the bone defect itself is not sufficient to cause the meningocele [2]. Other factors that influents are alterations in the brain and meningeal tissue as edema, cysts and hemorrhage, pressuring the herniation through the bone defect [3].

Often it can be misdiagnosed because there are only few characteristic symptoms. The most expected symptoms are: unilateral conductive hearing loss with middle (secondary to reduce ossicular mobility), ear effusion on at patients older than 40 years-old, headache, pulsatile tinnitus or cerebrospinal fluid leak [4], those symptoms must be specially alarming if patient underwent recent trauma or surgery. It can also be presented with recurrent episodes of meningitis, and rarely associated with epilepsy of the temporal lobe [3].

The diagnosis of the disease must consider the symptoms and signs numerated above combined with otoscopy, in which fluid transparent liquid can be found along with signs of accentuated convexity of the tympanic membrane, particularly of the pars flaccida. However, the colorless characteristic of the fluid can lead to a normal otoscopy diagnosis in most of the cases. An audiometry may also show a unilateral conductive hearing loss [4].

The gold standard for temporal meningocele and temporal bone defects diagnosis and treatment is the high-resolution tomography. MRI can be useful if encephalocele is established for planning reduction of brain mass. Other studies such as cisthernography and nuclear medicine studies are more invasive, due to lumbar puncture, 
and they do not change diagnoses or therapy compared with CT. $\beta_{2}$-transferrin tests can be done in cases of difficult diagnosis [4].

Temporal meningocele is an important pathology in otology and neurology practice for which surgery is required often. Closure of the defect can avoid meningitis, a potentially severe complication. In the other side, surgery has very few rates of complications [3].

The aim of this study is to describe the surgical approach of temporal meningocele and perform a medical literature review of this topic.

\section{Materials and Methods}

A clinical retrospective study of medical records of two patients who underwent surgical treatment for temporal bone meningocele.

Besides, a review of the medical literature in PubMed and Scopus database was performed, covering articles published since 1980 in Portuguese or English, using the following keywords (MeSH): "temporal bone meningocele, middle ear meningocele; hearing loss". The aim of this review was to identify the surgical techniques most commonly used for the treatment of this pathology and its clinical outcomes.

\section{Inclusion and exclusion criteria}

Only patients with confirmed diagnosis (clinical and radiological) of temporal bone meningocele and who underwent surgical treatment were included.

The medical records of the subjects were used in this review and detailed all the parameters of the surgical procedure and related devices.

\section{Ethics}

The institutional review board approved this study and all subjects gave written informed consent.

\section{Results}

\section{Case 1}

Female patient, 32 years old, presented with ear effusion five years after a mastoidectomy surgery due to cholesteatoma of the external auditory canal growing in direction to the mastoid bone. CT showed an image that suggested inner ear meningocele herniation, and patient underwent surgery. After surgery patient had an improvement of the audiometric tests, however she maintained ear effusion, and had to be submitted once again to a mastoidectomy revision due to cholesteatoma recurrence. At the present time patient has no ear effusion.

\section{Case 2}

Male patient, 29 years old, presented with ear effusion after cranial trauma 5 years before, submitted to surgery in 2004. After surgery patient maintained ear effusion and had two episodes of meningitis in 2005 and 2006, although cyntilography did not show meningocele. At 2006 patient underwent another surgery due residual meningocele that was identified and corrected, this time being asymptomatic post-surgery.

Intra operative findings and surgical approach showed no temporal bone fracture line and it was performed a modified radical mastoidectomy, canal up surgery, and all ossicular chain and tympanic membrane were preserved.

\section{Surgical approach}

The patient is placed on horizontal dorsal decubitus with the head turned to the contralateral side undergeneral anesthesia.

The operative field is prepared by ample trichotomy, antisepsis with $2 \%$ chlorhexidine, and placement of electrodes for monitoring the 8 th cranial nerve. The operative field is separated from the rest of the scalp by using micropore ${ }^{\mathrm{TM}}$. Antibiotic prophylaxis is done with endovenous cefazolin $(50 \mathrm{mg} / \mathrm{kg})$ during induction of anesthesia.

- Antisepsis with $0.2 \%$ aqueous chlorhexidine, placement of sterile drapes and steri-drape;

- Rectilinear retroauricular incision and dissection along anatomical planes.

- Removal of small fragments of fascia and temporal muscle;

- Resection of conchal cartilage.

- Simple mastoidectomy, identifying the lateral semicircular canal, the short ramus of the incus, the posterior wall of the outer ear canal, the tegmen timpani, and the lateral sinus;

- Dissection of the tegmen and visualization of the meningocele.

- Temporal muscle, cartilage and fascia along with fibrin glue is located. No other support was used.

- Closure with sutures on by planes and subcutaneous tissue; skin closure with Nylon 4.0;

- External compressive bandage.

In the postoperative time, the external compressive bandage was maintained for 72 hours and the patient is requested to rest and avoid abdominal's efforts/Valsalva. Figures $1 \mathrm{a}$ and b shows intraoperative images of case one, before and after meningocele correction. Both patients underwent this similar technique for correction of the meningocele, but few differences in mastoidectomy technique were made. Figures $2 \mathrm{a}$ and $\mathrm{b}$ shows computed tomography of ears and mastoid of case one pre surgery and post-surgery status. Long term fallow-up of 10 years showed no meningocele recurrence or middle ear effusion, and none of the patients presented new cases of meningitis.
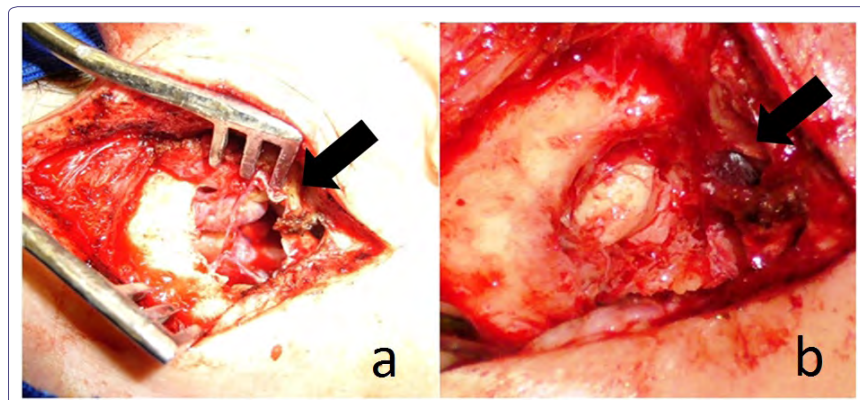

Figure 1: Intraoperative images of case one, arrow points to meningocele and tegmen defect corrected in image 2.

\section{Discussion}

Literature search showed only 9 publications/papers of temporal bone meningocele, in which 159 cases were described. Kong et al., [5] described a case of Trans labyrinthine meningocele with a vestibulocochlear malformation presenting as deafness and a middle ear mass, in which the treatment of meningocele was coverage with perichondrium and cartilage composite graft obtained from conchal cartilage to protect it from later trauma and to prevent CSF leakage [5]. 


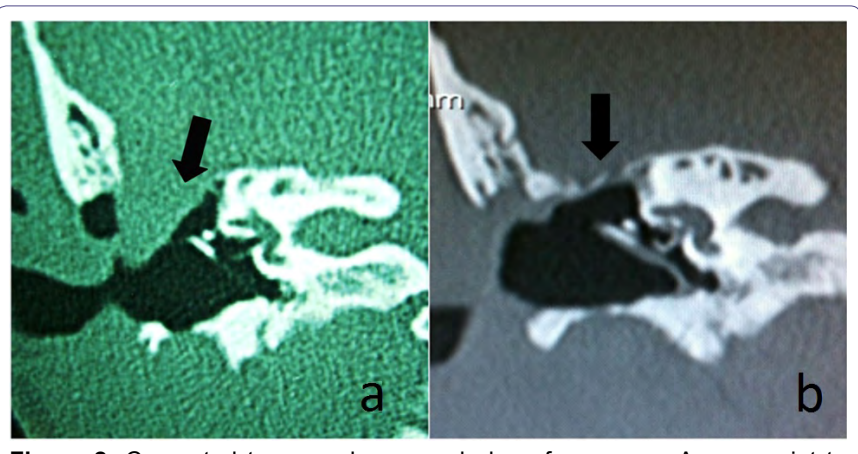

Figure 2: Computed tomography coronal plan of case one. Arrows point to pre-surgery status (3) and post-surgery status (4) of the tegmen with no residual meningocele.

Kasugai el al., [6] reported a case of meningocele who underwent repair surgery for the middle skull base defect with bone putty and covered by a pedunculated dermal flap of the temporal fascia after suturing the fistulous opening of duramater.

Kenning et al., [7] described a case series of 23 consecutive patients undergoing a combined mastoidectomy and middle cranial fossa craniotomy for the treatment of a tegmen defect. His technique involved a robust dural closure followed by extradural dissection and encephalocele reduction or resection. Both synthetic dural inlay and an epidural autologous graft along the tegmen were used to ensure that the areas of dural and osseous disruption are fully covered and reinforced [7].

Verma [8] described a case of meningocele that underwent mastoidectomy, followed by mass excision of the tumor, the bony defect was repaired in three tiers and superiorly wired mesh was placed covering the margins of the bony defect on all sides. This was followed by locally available conchal cartilage with intact mucoperichondrium on one side and then covered by a temporalis fascia graft tucked into the cranial and inferior side of the defect [8].

Mong et al., [9] described 2 cases of fallopian canal meningocele treated with combined transmastoid approach with middle fossa craniotomy, herniated tissue was amputated at the cranial base, and the defects were closed with condral cartilage, temporalis fascia, and middle turbinate mucosal-free grafts [9].

Savva et al., [10] published a series of 92 cases of temporal bone defects, in which 27 where traumatic cases and did not need surgery. The majority (79\%) required a transmastoid approach alone. Similarly, all non-traumatic (spontaneous) CSF leaks required surgical closure involving mastoidectomy or a combined mastoid and middle fossa approach. Repairs were less likely to fail if supporting material was used, and better success rates were achieved when a multilayer technique was used to support the initial closure [10].

Nahas et al., [11] reported a series of 15 patients with spontaneous temporal meningocele all treated with combined transmastoid-middle fossa approach. It presented a success rate of $100 \%$. Papanicolaou et al., [12] reported a case of meningocele treated with exploratory mastoidectomy, amputation of the herniated meningocele and closure of the defect with temporalis fascia and an inferiorly based pedunculated muscular flap [12].

Sergi et al., [13] reported 24 cases, all treated with transmastoid approach. In this study all cases had collagenous membrane used along with a bone graft, temporalis fascia and fibrin glue. No complications were demonstred in the medium 28 months follow-up [13].
We can notice from the literature that temporalis fascia along with condral cartilage are the most popular grafts, but mucosal flaps and other substances are often successful used to cover the bone defect.

We believe that there is much variability of the techniques presented, but there are some key points in common that may be responsible for the good successes presented by cited authors. A careful surgical dissection plus a definition of all meningocele, using some grafts and/or retails appears to be part of all the techniques presented.

In terms of surgical approach, the transmastoid technique has been shown safe and effective but finds its limitations according to the meningocele size. It seems that the most important factors considering success of the repair are multiple layers of supporting material as grafts, cartilage and others constituents.

Therefore, there are no significant differences between the kinds of repair material used, as long as the repair involves careful management and at least three kinds of different materials. Cartilage and fascia are a good example of effective and simple graft with great results in our patients and in the literature.

Finally, it is imperative to do a close follow-up to these patients controlling meningocele recurrence, hearing loss rehabilitation and risk factors control as cholesteatoma, weight gain, and trauma.

It is noteworthy that we found no description of techniques that had a surgical failure or need for rapprochement or further surgery.

\section{Conclusion}

It was presented excellent outcomes for solving a temporal meningocele using cartilage graft, temporal muscle and fibrin glue, with no complications.

\section{Competing Interests}

Nothing to declare. The author(s) declare that they have no competing interests

\section{References}

1. Sanna M, Fois P, Russo A, Falcioni M (2009) Management of meningoencephalic herniation of the temporal bone: Personal experience and literature review. Laryngoscope 119: 1579-1585.

2. Rao AK, Merenda DM, Wetmore SJ (2005) Diagnosis and management of spontaneous cerebrospinal fluid otorrhea. Otol Neurotol 26: 1171-1175.

3. Kim L, Wisely CE, Dodson EE (2014) Transmastoid approach to spontaneous temporal bone cerebrospinal fluid leaks: hearing improvement and success of repair. Otolaryngol Head Neck Surg 150: 472-478.

4. Alonso RC, de la Peña MJ, Caicoya AG, Rodriguez MR, Moreno EA, et al (2013) Spontaneous skull base meningoencephaloceles and cerebrospinal fluid fistulas. Radiographics 33: 553-570.

5. Kong WK, Lee $\mathrm{CH}$, Eunhye $\mathrm{Y}$, Shin SH (2014) Unruptured translabyrinthine meningocele without CSF otorrhea. Int J Pediatr Otorhinolaryngol 78: 566569.

6. Kasugai S, Kenmochi M, Nishino H, Okada T, Ohashi T (2003) A case of meningoencephalic herniation of the temporal bone. Auris Nasus Larynx 30: 103-105.

7. Kenning TJ, Willcox TO, Artz GJ, Schiffmacher P, Farrell CJ, et al. (2012) Surgical management of temporal meningoencephaloceles, cerebrospinal fluid leaks, and intracranial hypertension: treatment paradigm and outcomes. Neurosurg Focus 32: 6.

8. Verma R (2006) Meningoencephalocele of the middle ear cleft: Three tier management. Indian J Otolaryngol Head Neck Surg 58: 378-380.

9. Mong S, Goldberg AN, Lustig LR (2009) Fallopian canal meningocele: report of two cases. Otol Neurotol 30: 525-528. 
Citation: Toro MDC, Guimarães AC, de Carvalho GM, Paschoal JR, Pfeilsticker LN (2016) Transmastoid Cartilage Graft-Meningocele Temporal: Surgical Approach. J Otolaryng Head Neck Surg 2: 005.

- Page 4 of $4 \cdot$

10. Savva A, Taylor MJ, Beatty CW (2003) Management of cerebrospinal fluid leaks involving the temporal bone: report on 92 patients. Laryngoscope 113: 50-56.

11. Nahas Z, Tatlipinar A, Limb CJ, Francis HW (2008) Spontaneous meningoencephalocele of the temporal bone: clinical spectrum and presentation. Arch Otolaryngol Head Neck Surg 134: 509-518.
12. Papanikolaou V, Bibas A, Ferekidis E, Anagnostopoulou S, Xenellis J (2007) Idiopathic temporal bone encephalocele. Skull Base 17: 311-316.

13. Sergi B, Passali GC, Picciotti PM, De Corso E, Paludetti G (2013) Transmastoid approach to repair meningoencephalic herniation in the middle ear. Acta Otorhinolaryngol Ital 33: 97-101. 